\title{
Steuerentlastung bei eingeschränkter medizinischer Notwendigkeit
}

- Der Bundesfinanzhof (BFH) hat mit Urteil vom 2.9.2010 entschieden, dass Aufwendungen für eine immunbiologische Krebsabwehrtherapie steuerlich als außergewöhnliche Belastung abgezogen werden können. Damit hat er - in Abkehr von der bisherigen Rechtsprechung - anerkannt, dass auch Kosten für eine objektiv nicht zur Heilung oder Linderung geeignete Behandlung zwangsläufig erwachsen können, wenn eine Erkrankung mit einer nur noch begrenzten Lebenserwartung besteht, die nicht mehr auf eine kurative Behandlung anspricht.

Dies gilt selbst dann, wenn sich der Erkrankte für eine aus schulmedizinischer oder naturheilkundlicher Sicht nicht anerkannte Heilmethode entscheidet. Nicht die medizinische Notwendigkeit der Maßnahme begründe in diesen Fällen die tatsächliche Zwangsläufigkeit, sondern die Ausweglosigkeit der Lebenssituation, die den Griff nach jedem Strohhalm gebiete.

\section{MMW Kommentar}

Nach einem weiteren Urteil des Bundesfinanzhofs (BFH) vom 13.10.2010 sind auch die Kosten für einen krankheitsbedingten Aufenthalt in einem Seniorenheim als außergewöhnliche Belastung einkommensteuerlich abziehbar, wenn keine ständige Pflegebedürftigkeit besteht. Mit der Entscheidung rückt der BFH von seinen bisher strengeren Grundsätzen ab, wonach ein Abzug entweder zusätzliche Kosten für Pflegeleistungen oder die Ausstellung eines Schwerbehindertenausweises mit den Merkzeichen "H" oder "Bl"voraussetzte.

Im Urteilsfall war die damals 74-jährige Klägerin nach einer stationären Behandlung in einer psychiatrischen Klinik auf ärztliche Empfehlung in ein Seniorenheim gezogen. Ihre Wohnung in einem Zweifamilienhaus hatte sie währenddessen nicht aufgegeben. Das Finanzamt erkannte die geltend ge-
Dr. Gerd W. Zimmermann Facharzt für Allgemeinmedizin Kapellenstraße 9, D-65719 Hofheim

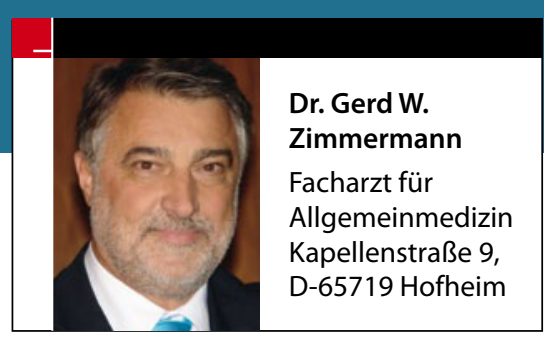

